\title{
Special Values of Generalized Log-sine Integrals
}

\author{
Jonathan M. Borwein \\ University of Newcastle \\ Callaghan, NSW 2308, Australia \\ jonathan.borwein@newcastle.edu.au
}

\author{
Armin Straub \\ Tulane University \\ New Orleans, LA 70118, USA \\ astraub@tulane.edu
}

\begin{abstract}
We study generalized log-sine integrals at special values. At $\pi$ and multiples thereof explicit evaluations are obtained in terms of Nielsen polylogarithms at \pm 1 . For general arguments we present algorithmic evaluations involving Nielsen polylogarithms at related arguments. In particular, we consider log-sine integrals at $\pi / 3$ which evaluate in terms of polylogarithms at the sixth root of unity. An implementation of our results for the computer algebra systems Mathematica and SAGE is provided.
\end{abstract}

\section{Categories and Subject Descriptors}

I.1.1 [Symbolic and Algebraic Manipulation]: Expressions and Their Representation; I.1.2 [Symbolic and Algebraic Manipulation]: Algorithms

\section{General Terms}

Algorithms, Theory

\section{Keywords}

log-sine integrals, multiple polylogarithms, multiple zeta values, Clausen functions

\section{INTRODUCTION}

For $n=1,2, \ldots$ and $k \geq 0$, we consider the (generalized) log-sine integrals defined by

$$
\operatorname{Ls}_{n}^{(k)}(\sigma):=-\int_{0}^{\sigma} \theta^{k} \log ^{n-1-k}\left|2 \sin \frac{\theta}{2}\right| \mathrm{d} \theta .
$$

The modulus is not needed for $0 \leq \sigma \leq 2 \pi$. For $k=0$ these are the (basic) log-sine integrals $\operatorname{Ls}_{n}(\sigma):=\operatorname{Ls}_{n}^{(0)}(\sigma)$. Various log-sine integral evaluations may be found in [20, $\S 7.6 \& \S 7.9]$.

In this paper, we will be concerned with evaluations of the log-sine integrals $\operatorname{Ls}_{n}^{(k)}(\sigma)$ for special values of $\sigma$. Such evaluations are useful for physics [15]: log-sine integrals appeared

Permission to make digital or hard copies of all or part of this work for personal or classroom use is granted without fee provided that copies are not made or distributed for profit or commercial advantage and that copies bear this notice and the full citation on the first page. To copy otherwise, to republish, to post on servers or to redistribute to lists, requires prior specific permission and/or a fee.

ISSAC'11, June 8-11, 2011, San Jose, California, USA

Copyright 2011 ACM 978-1-4503-0675-1/11/06 ...\$10.00. for instance in recent work on the $\varepsilon$-expansion of various Feynman diagrams in the calculation of higher terms in the $\varepsilon$-expansion, $[8,16,9,11,14]$. Of particular importance are the log-sine integrals at the special values $\pi / 3, \pi / 2,2 \pi / 3, \pi$. The log-sine integrals also appear in many settings in number theory and analysis: classes of inverse binomial sums can be expressed in terms of generalized log-sine integrals, [10, 4].

In Section 3 we focus on evaluations of log-sine and related integrals at $\pi$. General arguments are considered in Section 5 with a focus on the case $\pi / 3$ in Section 5.1. Imaginary arguments are briefly discussed in 5.2. The results obtained are suitable for implementation in a computer algebra system. Such an implementation is provided for Mathematica and SAGE, and is described in Section 7. This complements existing packages such as 1 sjk [15] for numerical evaluations of log-sine integrals or HPL [21] as well as [25] for working with multiple polylogarithms.

Further motivation for such evaluations was sparked by our recent study [6] of certain multiple Mahler measures. For $k$ functions (typically Laurent polynomials) in $n$ variables the multiple Mahler measure $\mu\left(P_{1}, P_{2}, \ldots, P_{k}\right)$, introduced in [18], is defined by

$$
\int_{0}^{1} \cdots \int_{0}^{1} \prod_{j=1}^{k} \log \left|P_{j}\left(e^{2 \pi i t_{1}}, \ldots, e^{2 \pi i t_{n}}\right)\right| \mathrm{d} t_{1} \mathrm{~d} t_{2} \ldots \mathrm{d} t_{n} .
$$

When $P=P_{1}=P_{2}=\cdots=P_{k}$ this devolves to a higher Mahler measure, $\mu_{k}(P)$, as introduced and examined in [18]. When $k=1$ both reduce to the standard (logarithmic) Mahler measure [7].

The multiple Mahler measure

$$
\mu_{k}\left(1+x+y_{*}\right):=\mu\left(1+x+y_{1}, 1+x+y_{2}, \ldots, 1+x+y_{k}\right)
$$

was studied by Sasaki [24, Lemma 1] who provided an evaluation of $\mu_{2}\left(1+x+y_{*}\right)$. It was observed in [6] that

$$
\mu_{k}\left(1+x+y_{*}\right)=\frac{1}{\pi} \operatorname{Ls}_{k+1}\left(\frac{\pi}{3}\right)-\frac{1}{\pi} \operatorname{Ls}_{k+1}(\pi) .
$$

Many other Mahler measures studied in $[6,1]$ were shown to have evaluations involving generalized log-sine integrals at $\pi$ and $\pi / 3$ as well.

To our knowledge, this is the most exacting such study undertaken - perhaps because it would be quite impossible without modern computational tools and absent a use of the quite recent understanding of multiple polylogarithms and multiple zeta values [3]. 


\section{PRELIMINARIES}

In the following, we will denote the multiple polylogarithm as studied for instance in [4] and [2, Ch. 3] by

$$
\operatorname{Li}_{a_{1}, \ldots, a_{k}}(z):=\sum_{n_{1}>\cdots>n_{k}>0} \frac{z^{n_{1}}}{n_{1}^{a_{1}} \cdots n_{k}^{a_{k}}} .
$$

For our purposes, the $a_{1}, \ldots, a_{k}$ will usually be positive integers and $a_{1} \geq 2$ so that the sum converges for all $|z| \leq 1$. For example, $\operatorname{Li}_{2,1}(z)=\sum_{k=1}^{\infty} \frac{z^{k}}{k^{2}} \sum_{j=1}^{k-1} \frac{1}{j}$. In particular, $\operatorname{Li}_{k}(x):=\sum_{n=1}^{\infty} \frac{x^{n}}{n^{k}}$ is the polylogarithm of order $k$. The usual notation will be used for repetitions so that, for instance, $\operatorname{Li}_{2,\{1\}^{3}}(z)=\operatorname{Li}_{2,1,1,1}(z)$.

Moreover, multiple zeta values are denoted by

$$
\zeta\left(a_{1}, \ldots, a_{k}\right):=\operatorname{Li}_{a_{1}, \ldots, a_{k}}(1) .
$$

Similarly, we consider the multiple Clausen functions $(\mathrm{Cl})$ and multiple Glaisher functions (Gl) of depth $k$ and weight $w=a_{1}+\ldots+a_{k}$ defined as

$$
\begin{aligned}
& \mathrm{Cl}_{a_{1}, \ldots, a_{k}}(\theta)=\left\{\begin{array}{ll}
\operatorname{Im}_{\operatorname{Li}_{a_{1}, \ldots, a_{k}}\left(e^{i \theta}\right)} & \text { if } w \text { even } \\
\operatorname{Re}_{\operatorname{Li}_{a_{1}, \ldots, a_{k}}\left(e^{i \theta}\right)} & \text { if } w \text { odd }
\end{array}\right\},
\end{aligned}
$$

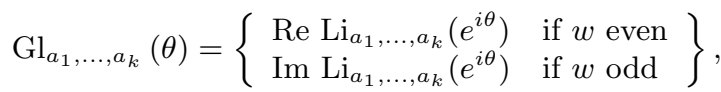

in accordance with [20]. Of particular importance will be the case of $\theta=\pi / 3$ which has also been considered in [4].

Our other notation and usage is largely consistent with that in [20] and that in the newly published [23] in which most of the requisite material is described. Finally, a recent elaboration of what is meant when we speak about evaluations and "closed forms" is to be found in [5].

\section{EVALUATIONS AT $\pi$}

\subsection{Basic log-sine integrals at $\pi$}

The exponential generating function, [19, 20],

$$
-\frac{1}{\pi} \sum_{m=0}^{\infty} \operatorname{Ls}_{m+1}(\pi) \frac{\lambda^{m}}{m !}=\frac{\Gamma(1+\lambda)}{\Gamma^{2}\left(1+\frac{\lambda}{2}\right)}=\left(\begin{array}{c}
\lambda \\
\frac{\lambda}{2}
\end{array}\right)
$$

is well-known and implies the recurrence

$$
\begin{aligned}
& \frac{(-1)^{n}}{n !} \operatorname{Ls}_{n+2}(\pi)=\pi \alpha(n+1) \\
& \quad+\sum_{k=1}^{n-2} \frac{(-1)^{k}}{(k+1) !} \alpha(n-k) \operatorname{Ls}_{k+2}(\pi),
\end{aligned}
$$

where $\alpha(m)=\left(1-2^{1-m}\right) \zeta(m)$.

Example 1. (Values of $\operatorname{Ls}_{n}(\pi)$ ) We have $\operatorname{Ls}_{2}(\pi)=0$ and

$$
\begin{aligned}
-\operatorname{Ls}_{3}(\pi) & =\frac{1}{12} \pi^{3} \\
\operatorname{Ls}_{4}(\pi) & =\frac{3}{2} \pi \zeta(3) \\
-\operatorname{Ls}_{5}(\pi) & =\frac{19}{240} \pi^{5} \\
\operatorname{Ls}_{6}(\pi) & =\frac{45}{2} \pi \zeta(5)+\frac{5}{4} \pi^{3} \zeta(3) \\
-\operatorname{Ls}_{7}(\pi) & =\frac{275}{1344} \pi^{7}+\frac{45}{2} \pi \zeta(3)^{2} \\
\operatorname{Ls}_{8}(\pi) & =\frac{2835}{4} \pi \zeta(7)+\frac{315}{8} \pi^{3} \zeta(5)+\frac{133}{32} \pi^{5} \zeta(3),
\end{aligned}
$$

and so forth. The fact that each integral is a multivariable rational polynomial in $\pi$ and zeta values follows directly from the recursion (7). Alternatively, these values may be conveniently obtained from (6) by a computer algebra system. For instance, in Mathematica the code

FullSimplify $[D[-$ Binomial $[\mathrm{x}, \mathrm{x} / 2],\{\mathrm{x}, 6\}] / . \mathrm{x}->0]$

produces the above evaluation of $\operatorname{Ls}_{6}(\pi)$.

\subsection{The log-sine-cosine integrals}

The log-sine-cosine integrals

$$
\operatorname{Lsc}_{m, n}(\sigma):=-\int_{0}^{\sigma} \log ^{m-1}\left|2 \sin \frac{\theta}{2}\right| \log ^{n-1}\left|2 \cos \frac{\theta}{2}\right| \mathrm{d} \theta
$$

appear in physical applications as well, see for instance $[9$, 14]. They have also been considered by Lewin, [19, 20], and he demonstrates how their values at $\sigma=\pi$ may be obtained much the same as those of the log-sine integrals in Section 3.1. As observed in [1], Lewin's result can be put in the form

$$
\begin{gathered}
-\frac{1}{\pi} \sum_{m, n=0}^{\infty} \operatorname{Lsc}_{m+1, n+1}(\pi) \frac{x^{m}}{m !} \frac{y^{n}}{n !}=\frac{2^{x+y}}{\pi} \frac{\Gamma\left(\frac{1+x}{2}\right) \Gamma\left(\frac{1+y}{2}\right)}{\Gamma\left(1+\frac{x+y}{2}\right)} \\
=\left(\begin{array}{c}
x \\
x / 2
\end{array}\right)\left(\begin{array}{c}
y \\
y / 2
\end{array}\right) \frac{\Gamma\left(1+\frac{x}{2}\right) \Gamma\left(1+\frac{y}{2}\right)}{\Gamma\left(1+\frac{x+y}{2}\right)} .
\end{gathered}
$$

The last form makes it clear that this is an extension of (6).

The notation Lsc has been introduced in [9] where evaluations for other values of $\sigma$ and low weight can be found.

\subsection{Log-sine integrals at $\pi$}

As Lewin [20, §7.9] sketches, at least for small values of $n$ and $k$, the generalized log-sine integrals $\operatorname{Ls}_{n}^{(k)}(\pi)$ have closed forms involving zeta values and Kummer-type constants such as $\mathrm{Li}_{4}(1 / 2)$. This will be made more precise in Remark 1 . Our analysis starts with the generating function identity

$$
\begin{aligned}
& -\sum_{n, k \geq 0} \operatorname{Ls}_{n+k+1}^{(k)}(\pi) \frac{\lambda^{n}}{n !} \frac{(i \mu)^{k}}{k !}=\int_{0}^{\pi}\left(2 \sin \frac{\theta}{2}\right)^{\lambda} \mathrm{e}^{i \mu \theta} \mathrm{d} \theta \\
& =i \mathrm{e}^{i \pi \frac{\lambda}{2}} B_{1}\left(\mu-\frac{\lambda}{2}, 1+\lambda\right)-i \mathrm{e}^{i \pi \mu} B_{1 / 2}\left(\mu-\frac{\lambda}{2},-\mu-\frac{\lambda}{2}\right)
\end{aligned}
$$

given in [20]. Here $B_{x}$ is the incomplete Beta function:

$$
B_{x}(a, b)=\int_{0}^{x} t^{a-1}(1-t)^{b-1} \mathrm{~d} t .
$$

We shall show that with care - because of the singularities at zero - (10) can be differentiated as needed as suggested by Lewin.

Using the identities, valid for $a, b>0$ and $0<x<1$,

$$
\begin{aligned}
B_{x}(a, b) & =\frac{x^{a}(1-x)^{b-1}}{a}{ }_{2} F_{1}\left(\begin{array}{c}
1-b, 1 \\
a+1
\end{array} \mid \frac{x}{x-1}\right) \\
& =\frac{x^{a}(1-x)^{b}}{a}{ }_{2} F_{1}\left(\begin{array}{c}
a+b, 1 \\
a+1
\end{array} \mid x\right),
\end{aligned}
$$

found for instance in $[23, \S 8.17(\mathrm{ii})]$, the generating function (10) can be rewritten as

$$
i \mathrm{e}^{i \pi \frac{\lambda}{2}}\left(B_{1}\left(\mu-\frac{\lambda}{2}, 1+\lambda\right)-B_{-1}\left(\mu-\frac{\lambda}{2}, 1+\lambda\right)\right) .
$$

Upon expanding this we obtain the following computationally more accessible generating function for $\operatorname{Ls}_{n+k+1}^{(k)}(\pi)$ : 
TheOREM 1. For $2|\mu|<\lambda<1$ we have

$$
\begin{aligned}
-\sum_{n, k \geq 0} \operatorname{Ls}_{n+k+1}^{(k)}(\pi) \frac{\lambda^{n}}{n !} \frac{(i \mu)^{k}}{k !} \\
=i \sum_{n \geq 0}\left(\begin{array}{l}
\lambda \\
n
\end{array}\right) \frac{(-1)^{n} \mathrm{e}^{i \pi \frac{\lambda}{2}}-\mathrm{e}^{i \pi \mu}}{\mu-\frac{\lambda}{2}+n} .
\end{aligned}
$$

We now show how the log-sine integrals $\operatorname{Ls}_{n}^{(k)}(\pi)$ can quite comfortably be extracted from (11) by differentiating its right-hand side. The case $n=0$ is covered by:

Proposition 1. We have

$$
\left.\frac{\mathrm{d}^{k}}{\mathrm{~d} \mu^{k}} \frac{\mathrm{d}^{m}}{\mathrm{~d} \lambda^{m}} i \frac{\mathrm{e}^{i \pi \frac{\lambda}{2}}-\mathrm{e}^{i \pi \mu}}{\mu-\frac{\lambda}{2}}\right|_{\substack{\lambda=0 \\ \mu=0}}=\frac{\pi}{2^{m}}(i \pi)^{m+k} B(m+1, k+1) .
$$

Proof. This may be deduced from

$$
\begin{aligned}
\frac{\mathrm{e}^{x}-\mathrm{e}^{y}}{x-y} & =\sum_{m, k \geq 0} \frac{x^{m} y^{k}}{(k+m+1) !} \\
& =\sum_{m, k \geq 0} B(m+1, k+1) \frac{x^{m}}{m !} \frac{y^{k}}{k !}
\end{aligned}
$$

upon setting $x=i \pi \lambda / 2$ and $y=i \pi \mu$.

The next proposition is most helpful in differentiation of the right-hand side of (11) for $n \geq 1$, Here, we denote a multiple harmonic number by

$$
H_{n-1}^{[\alpha]}:=\sum_{n>i_{1}>i_{2}>\ldots>i_{\alpha}} \frac{1}{i_{1} i_{2} \cdots i_{\alpha}} .
$$

If $\alpha=0$ we set $H_{n-1}^{[0]}:=1$.

Proposition 2. For $n \geq 1$

$$
\left.\frac{(-1)^{\alpha}}{\alpha !}\left(\frac{\mathrm{d}}{\mathrm{d} \lambda}\right)^{\alpha}\left(\begin{array}{l}
\lambda \\
n
\end{array}\right)\right|_{\lambda=0}=\frac{(-1)^{n}}{n} H_{n-1}^{[\alpha-1]} .
$$

Note that, for $\alpha \geq 0$,

$$
\sum_{n \geq 0} \frac{( \pm 1)^{n}}{n^{\beta}} H_{n-1}^{[\alpha]}=\operatorname{Li}_{\beta,\{1\}^{\alpha}}( \pm 1)
$$

which shows that the evaluation of the log-sine integrals will involve Nielsen polylogarithms at \pm 1 , that is polylogarithms of the type $\mathrm{Li}_{a,\{1\}^{b}}( \pm 1)$.

Using the Leibniz rule coupled with Proposition 2 to differentiate (11) for $n \geq 1$ and Proposition 1 in the case $n=0$, it is possible to explicitly write $\operatorname{Ls}_{n}^{(k)}(\pi)$ as a finite sum of Nielsen polylogarithms with coefficients only being rational multiples of powers of $\pi$. The process is now exemplified for $\operatorname{Ls}_{4}^{(2)}(\pi)$ and $\operatorname{Ls}_{5}^{(1)}(\pi)$.

Example 2. $\left(\mathrm{Ls}_{4}^{(2)}(\pi)\right)$ To find $\mathrm{Ls}_{4}^{(2)}(\pi)$ we differentiate (11) once with respect to $\lambda$ and twice with respect to $\mu$. To simplify computation, we exploit the fact that the result will be real which allows us to neglect imaginary parts:

$$
\begin{aligned}
-\mathrm{Ls}_{4}^{(2)}(\pi) & =\left.\frac{\mathrm{d}^{2}}{\mathrm{~d} \mu^{2}} \frac{\mathrm{d}}{\mathrm{d} \lambda} i \sum_{n \geq 0}\left(\begin{array}{l}
\lambda \\
n
\end{array}\right) \frac{(-1)^{n} \mathrm{e}^{i \pi \frac{\lambda}{2}}-\mathrm{e}^{i \pi \mu}}{\mu-\frac{\lambda}{2}+n}\right|_{\lambda=\mu=0} \\
& =2 \pi \sum_{n \geq 1} \frac{(-1)^{n+1}}{n^{3}}=\frac{3}{2} \pi \zeta(3) .
\end{aligned}
$$

In the second step we were able to drop the term corresponding to $n=0$ because its contribution $-i \pi^{4} / 24$ is purely imaginary as follows a priori from Proposition 2.

Example 3. $\left(\operatorname{Ls}_{5}^{(1)}(\pi)\right)$ Similarly, setting

$$
\mathrm{Li}_{a_{1}, \ldots, a_{n}}^{ \pm}:=\mathrm{Li}_{a_{1}, \ldots, a_{n}}(1)-\mathrm{Li}_{a_{1}, \ldots, a_{n}}(-1)
$$

we obtain $\operatorname{Ls}_{5}^{(1)}(\pi)$ as

$$
\begin{aligned}
-\mathrm{Ls}_{5}^{(1)}(\pi)= & \frac{3}{4} \sum_{n \geq 1} \frac{8\left(1-(-1)^{n}\right)}{n^{4}}\left(n H_{n-1}^{[2]}-H_{n-1}\right) \\
& +\frac{6\left(1-(-1)^{n}\right)}{n^{5}}-\frac{\pi^{2}}{n^{3}} \\
= & 6 \mathrm{Li}_{3,1,1}^{ \pm}-6 \mathrm{Li}_{4,1}^{ \pm}+\frac{9}{2} \mathrm{Li}_{5}^{ \pm}-\frac{3}{4} \pi^{2} \zeta(3) \\
= & -6 \mathrm{Li}_{3,1,1}(-1)+\frac{105}{32} \zeta(5)-\frac{1}{4} \pi^{2} \zeta(3) .
\end{aligned}
$$

The last form is what is automatically produced by our program, see Example 13, and is obtained from the previous expression by reducing the polylogarithms as discussed in Section 6.

The next example hints at the rapidly growing complexity of these integrals, especially when compared to the evaluations given in Examples 2 and 3.

Example 4. $\left(\operatorname{Ls}_{6}^{(1)}(\pi)\right)$ Proceeding as before we find

$$
\begin{aligned}
-\mathrm{Ls}_{6}^{(1)}(\pi)= & -24 \mathrm{Li}_{3,1,1,1}^{ \pm}+24 \mathrm{Li}_{4,1,1}^{ \pm}-18 \mathrm{Li}_{5,1}^{ \pm}+12 \mathrm{Li}_{6}^{ \pm} \\
& +3 \pi^{2} \zeta(3,1)-3 \pi^{2} \zeta(4)+\frac{\pi^{6}}{480} \\
= & 24 \mathrm{Li}_{3,1,1,1}(-1)-18 \mathrm{Li}_{5,1}(-1) \\
& +3 \zeta(3)^{2}-\frac{3}{1120} \pi^{6}
\end{aligned}
$$

In the first equality, the term $\pi^{6} / 480$ is the one corresponding to $n=0$ in (11) obtained from Proposition 1. The second form is again the automatically reduced output of our program.

Remark 1. From the form of (11) and (13) we find that the log-sine integrals $\operatorname{Ls}_{n}^{(k)}(\pi)$ can be expressed in terms of $\pi$ and Nielsen polylogarithms at \pm 1 . Using the duality results in $[3, \S 6.3$, and Example 2.4] the polylogarithms at -1 may be explicitly reexpressed as multiple polylogarithms at $1 / 2$. Some examples are given in [6].

Particular cases of Theorem 1 have been considered in [15] where explicit formulae are given for $\operatorname{Ls}_{n}^{(k)}(\pi)$ where $k=0,1,2$.

\subsection{Log-sine integrals at $2 \pi$}

As observed by Lewin [20, 7.9.8], log-sine integrals at $2 \pi$ are expressible in terms of zeta values only. If we proceed as in the case of evaluations at $\pi$ in (10) we find that the resulting integral now becomes expressible in terms of gamma functions:

$$
\begin{aligned}
-\sum_{n, k \geq 0} \operatorname{Ls}_{n+k+1}^{(k)}(2 \pi) \frac{\lambda^{n}}{n !} \frac{(i \mu)^{k}}{k !} & =\int_{0}^{2 \pi}\left(2 \sin \frac{\theta}{2}\right)^{\lambda} \mathrm{e}^{i \mu \theta} \mathrm{d} \theta \\
& =2 \pi \mathrm{e}^{i \mu \pi}\left(\begin{array}{c}
\lambda \\
\frac{\lambda}{2}+\mu
\end{array}\right)
\end{aligned}
$$


The special case $\mu=0$, in the light of (20) which gives $\operatorname{Ls}_{n}(2 \pi)=2 \operatorname{Ls}_{n}(\pi)$, recovers $(6)$.

We may now extract log-sine integrals $\operatorname{Ls}_{n}^{(k)}(2 \pi)$ in a similar way as described in Section 3.1.

Example 5. For instance,

$$
\operatorname{Ls}_{5}^{(2)}(2 \pi)=-\frac{13}{45} \pi^{5}
$$

We remark that this evaluation is incorrectly given in $[20$, (7.144)] as $7 \pi^{5} / 30$ underscoring an advantage of automated evaluations over tables (indeed, there are more misprints in [20] pointed out for instance in $[9,15])$.

\subsection{Log-sine-polylog integrals}

Motivated by the integrals $\operatorname{LsLsc}_{k, i, j}$ defined in [14] we show that the considerations of Section 3.3 can be extended to more involved integrals including

$$
\operatorname{Ls}_{n}^{(k)}(\pi ; d):=-\int_{0}^{\pi} \theta^{k} \log ^{n-k-1}\left(2 \sin \frac{\theta}{2}\right) \operatorname{Li}_{d}\left(\mathrm{e}^{i \theta}\right) \mathrm{d} \theta .
$$

On expressing $\operatorname{Li}_{d}\left(\mathrm{e}^{i \theta}\right)$ as a series, rearranging, and applying Theorem 1, we obtain the following exponential generating function for $\operatorname{Ls}_{n}^{(k)}(\pi ; d)$ :

Corollary 1. For $d \geq 0$ we have

$$
\begin{aligned}
-\sum_{n, k \geq 0} & \operatorname{Ls}_{n+k+1}^{(k)}(\pi ; d) \frac{\lambda^{n}}{n !} \frac{(i \mu)^{k}}{k !} \\
& =i \sum_{n \geq 1} H_{n, d}(\lambda) \frac{\mathrm{e}^{i \pi \frac{\lambda}{2}}-(-1)^{n} \mathrm{e}^{i \pi \mu}}{\mu-\frac{\lambda}{2}+n}
\end{aligned}
$$

where

$$
H_{n, d}(\lambda):=\sum_{k=0}^{n-1} \frac{(-1)^{k}\left(\begin{array}{l}
\lambda \\
k
\end{array}\right)}{(n-k)^{d}}
$$

We note for $0 \leq \theta \leq \pi$ that $\operatorname{Li}_{-1}\left(\mathrm{e}^{i \theta}\right)=-1 /\left(2 \sin \frac{\theta}{2}\right)^{2}$, $\operatorname{Li}_{0}\left(\mathrm{e}^{i \theta}\right)=-\frac{1}{2}+\frac{i}{2} \cot \frac{\theta}{2}$, while $\operatorname{Li}_{1}\left(\mathrm{e}^{i \theta}\right)=-\log \left(2 \sin \frac{\theta}{2}\right)+$ $i \frac{\pi-\theta}{2}$, and $\operatorname{Li}_{2}\left(\mathrm{e}^{i \theta}\right)=\zeta(2)+\frac{\theta}{2}\left(\frac{\theta}{2}-\pi\right)+i \mathrm{Cl}_{2}(\theta)$.

Remark 2. Corresponding results for an arbitrary Dirichlet series $\mathrm{L}_{\mathbf{a}, d}(x):=\sum_{n \geq 1} a_{n} x^{n} / n^{d}$ can be easily derived in the same fashion. Indeed, for

$$
\operatorname{Ls}_{n}^{(k)}(\pi ; \mathbf{a}, d):=-\int_{0}^{\pi} \theta^{k} \log ^{n-k-1}\left(2 \sin \frac{\theta}{2}\right) \mathrm{L}_{\mathbf{a}, d}\left(\mathrm{e}^{i \theta}\right) \mathrm{d} \theta
$$

one derives the exponential generating function (16) with $H_{n, d}(\lambda)$ replaced by

$$
H_{n, \mathbf{a}, d}(\lambda):=\sum_{k=0}^{n-1} \frac{(-1)^{k}\left(\begin{array}{l}
\lambda \\
k
\end{array}\right) a_{n-k}}{(n-k)^{d}} .
$$

This allows for $\operatorname{Ls}_{n}^{(k)}(\pi ; \mathbf{a}, d)$ to be extracted for many number theoretic functions. It does not however seem to cover any of the values of the $\operatorname{LsLsc}_{k, i, j}$ function defined in [14] that are not already covered by Corollary 1 .

\section{QUASIPERIODIC PROPERTIES}

As shown in $[20,(7.1 .24)]$, it follows from the periodicity of the integrand that, for integers $m$,

$$
\begin{aligned}
\operatorname{Ls}_{n}^{(k)}(2 m \pi) & -\operatorname{Ls}_{n}^{(k)}(2 m \pi-\sigma) \\
& =\sum_{j=0}^{k}(-1)^{k-j}(2 m \pi)^{j}\left(\begin{array}{l}
k \\
j
\end{array}\right) \operatorname{Ls}_{n-j}^{(k-j)}(\sigma) .
\end{aligned}
$$

Based on this quasiperiodic property of the log-sine integrals, the results of Section 3.4 easily generalize to show that logsine integrals at multiples of $2 \pi$ evaluate in terms of zeta values. This is shown in Section 4.1. It then follows from (19) that log-sine integrals at general arguments can be reduced to log-sine integrals at arguments $0 \leq \sigma \leq \pi$. This is discussed briefly in Section 4.2.

Example 6. In the case $k=0$, we have that

$$
\operatorname{Ls}_{n}(2 m \pi)=2 m \operatorname{Ls}_{n}(\pi) .
$$

For $k=1$, specializing (19) to $\sigma=2 m \pi$ then yields

$$
\operatorname{Ls}_{n}^{(1)}(2 m \pi)=2 m^{2} \pi \operatorname{Ls}_{n-1}(\pi)
$$

as is given in $[20,(7.1 .23)]$.

\subsection{Log-sine integrals at multiples of $2 \pi$}

For odd $k$, specializing (19) to $\sigma=2 m \pi$, we find

$$
2 \operatorname{Ls}_{n}^{(k)}(2 m \pi)=\sum_{j=1}^{k}(-1)^{j-1}(2 m \pi)^{j}\left(\begin{array}{l}
k \\
j
\end{array}\right) \operatorname{Ls}_{n-j}^{(k-j)}(2 m \pi)
$$

giving $\operatorname{Ls}_{n}^{(k)}(2 m \pi)$ in terms of lower order log-sine integrals.

More generally, on setting $\sigma=2 \pi$ in (19) and summing the resulting equations for increasing $m$ in a telescoping fashion, we arrive at the following reduction. We will use the standard notation

$$
H_{n}^{(a)}:=\sum_{k=1}^{n} k^{-a}
$$

for generalized harmonic sums.

Theorem 2. For integers $m \geq 0$,

$$
\operatorname{Ls}_{n}^{(k)}(2 m \pi)=\sum_{j=0}^{k}(-1)^{k-j}(2 \pi)^{j}\left(\begin{array}{l}
k \\
j
\end{array}\right) H_{m}^{(-j)} \operatorname{Ls}_{n-j}^{(k-j)}(2 \pi) \text {. }
$$

Summarizing, we have thus shown that the generalized log-sine integrals at multiples of $2 \pi$ may always be evaluated in terms of integrals at $2 \pi$. In particular, $\mathrm{Ls}_{n}^{(k)}(2 m \pi)$ can always be evaluated in terms of zeta values by the methods of Section 3.4.

\subsection{Reduction of arguments}

A general (real) argument $\sigma$ can be written uniquely as $\sigma=2 m \pi \pm \sigma_{0}$ where $m \geq 0$ is an integer and $0 \leq \sigma_{0} \leq \pi$. It then follows from (19) and

$$
\operatorname{Ls}_{n}^{(k)}(-\theta)=(-1)^{k+1} \operatorname{Ls}_{n}^{(k)}(\theta)
$$

that $\operatorname{Ls}_{n}^{(k)}(\sigma)$ equals

$$
\operatorname{Ls}_{n}^{(k)}(2 m \pi) \pm \sum_{j=0}^{k}( \pm 1)^{k-j}(2 m \pi)^{j}\left(\begin{array}{l}
k \\
j
\end{array}\right) \operatorname{Ls}_{n-j}^{(k-j)}\left(\sigma_{0}\right) .
$$


Since the evaluation of log-sine integrals at multiples of $2 \pi$ was explicitly treated in Section 4.1 this implies that the evaluation of log-sine integrals at general arguments $\sigma$ reduces to the case of arguments $0 \leq \sigma \leq \pi$.

\section{EVALUATIONS AT OTHER VALUES}

In this section we first discuss a method for evaluating the generalized log-sine integrals at arbitrary arguments in terms of Nielsen polylogarithms at related arguments. The gist of our technique originates with Fuchs ([12], [20, §7.10]). Related evaluations appear in [8] for $\operatorname{Ls}_{3}(\tau)$ to $\operatorname{Ls}_{6}(\tau)$ as well as in [9] for $\operatorname{Ls}_{n}(\tau)$ and $\operatorname{Ls}_{n}^{(1)}(\tau)$.

We then specialize to evaluations at $\pi / 3$ in Section 5.1. The polylogarithms arising in this case have been studied under the name of multiple Clausen and Glaisher values in [4]. In fact, the next result (22) with $\tau=\pi / 3$ is a modified version of $[4$, Lemma 3.2]. We employ the notation

$$
\left(\begin{array}{c}
n \\
a_{1}, \ldots, a_{k}
\end{array}\right):=\frac{n !}{a_{1} ! \cdots a_{k} !\left(n-a_{1}-\ldots-a_{k}\right) !}
$$

for multinomial coefficients.

THEOREM 3. For $0 \leq \tau \leq 2 \pi$, and nonnegative integers $n, k$ such that $n-k \geq 2$,

$$
\begin{aligned}
\zeta(n- & \left.k,\{1\}^{k}\right)-\sum_{j=0}^{k} \frac{(-i \tau)^{j}}{j !} \operatorname{Li}_{2+k-j,\{1\}^{n-k-2}}\left(\mathrm{e}^{i \tau}\right) \\
= & \frac{i^{k+1}(-1)^{n-1}}{(n-1) !} \sum_{r=0}^{n-k-1} \sum_{m=0}^{r}\left(\begin{array}{c}
n-1 \\
k, m, r-m
\end{array}\right) \\
& \times\left(\frac{i}{2}\right)^{r}(-\pi)^{r-m} \operatorname{Ls}_{n-(r-m)}^{(k+m)}(\tau) .
\end{aligned}
$$

Proof. Starting with

$$
\operatorname{Li}_{k,\{1\}^{n}}(\alpha)-\operatorname{Li}_{k,\{1\}^{n}}(1)=\int_{1}^{\alpha} \frac{\operatorname{Li}_{k-1,\{1\}^{n}}(z)}{z} \mathrm{~d} z
$$

and integrating by parts repeatedly, we obtain

$$
\begin{aligned}
& \sum_{j=0}^{k-2} \frac{(-1)^{j}}{j !} \log ^{j}(\alpha) \operatorname{Li}_{k-j,\{1\}^{n}}(\alpha)-\operatorname{Li}_{k,\{1\}^{n}}(1) \\
& \quad=\frac{(-1)^{k-2}}{(k-2) !} \int_{1}^{\alpha} \frac{\log ^{k-2}(z) \operatorname{Li}_{\{1\}^{n+1}}(z)}{z} \mathrm{~d} z .
\end{aligned}
$$

Letting $\alpha=\mathrm{e}^{i \tau}$ and changing variables to $z=\mathrm{e}^{i \theta}$, as well as using

$$
\operatorname{Li}_{\{1\}^{n}}(z)=\frac{(-\log (1-z))^{n}}{n !}
$$

the right-hand side of (23) can be rewritten as

$$
\frac{(-1)^{k-2}}{(k-2) !} \frac{i}{(n+1) !} \int_{0}^{\tau}(i \theta)^{k-2}\left(-\log \left(1-\mathrm{e}^{i \theta}\right)\right)^{n+1} \mathrm{~d} \theta .
$$

Since, for $0 \leq \theta \leq 2 \pi$ and the principal branch of the logarithm,

$$
\log \left(1-\mathrm{e}^{i \theta}\right)=\log \left|2 \sin \frac{\theta}{2}\right|+\frac{i}{2}(\theta-\pi),
$$

this last integral can now be expanded in terms of generalized log-sine integrals at $\tau$.

$$
\begin{aligned}
& \zeta\left(k,\{1\}^{n}\right)- \sum_{j=0}^{k-2} \frac{(-i \tau)^{j}}{j !} \operatorname{Li}_{k-j,\{1\}^{n}}\left(\mathrm{e}^{i \tau}\right) \\
&= \frac{(-i)^{k-1}}{(k-2) !} \frac{(-1)^{n}}{(n+1) !} \sum_{r=0}^{n+1} \sum_{m=0}^{r}\left(\begin{array}{c}
n+1 \\
r
\end{array}\right)\left(\begin{array}{c}
r \\
m
\end{array}\right) \\
&\left(\frac{i}{2}\right)^{r}(-\pi)^{r-m} \operatorname{Ls}_{n+k-(r-m)}^{(k+m-2)}(\tau) .
\end{aligned}
$$

Applying the $M Z V$ duality formula [3], we have

$$
\zeta\left(k,\{1\}^{n}\right)=\zeta\left(n+2,\{1\}^{k-2}\right),
$$

and a change of variables yields the claim.

We recall that the real and imaginary parts of the multiple polylogarithms are Clausen and Glaisher functions as defined in (4) and (5).

Example 7. Applying (22) with $n=4$ and $k=1$ and solving for $\mathrm{Ls}_{4}^{(1)}(\tau)$ yields

$$
\begin{aligned}
\mathrm{Ls}_{4}^{(1)}(\tau) & =2 \zeta(3,1)-2 \mathrm{Gl}_{3,1}(\tau)-2 \tau \mathrm{Gl}_{2,1}(\tau) \\
& +\frac{1}{4} \mathrm{Ls}_{4}^{(3)}(\tau)-\frac{1}{2} \pi \mathrm{Ls}_{3}^{(2)}(\tau)+\frac{1}{4} \pi^{2} \mathrm{Ls}_{2}^{(1)}(\tau) \\
& =\frac{1}{180} \pi^{4}-2 \mathrm{Gl}_{3,1}(\tau)-2 \tau \mathrm{Gl}_{2,1}(\tau) \\
& -\frac{1}{16} \tau^{4}+\frac{1}{6} \pi \tau^{3}-\frac{1}{8} \pi^{2} \tau^{2}
\end{aligned}
$$

For the last equality we used the trivial evaluation

$$
\operatorname{Ls}_{n}^{(n-1)}(\tau)=-\frac{\tau^{n}}{n}
$$

It appears that both $\mathrm{Gl}_{2,1}(\tau)$ and $\mathrm{Gl}_{3,1}(\tau)$ are not reducible for $\tau=\pi / 2$ or $\tau=2 \pi / 3$. Here, reducible means expressible in terms of multi zeta values and Glaisher functions of the same argument and lower weight. In the case $\tau=\pi / 3$ such reductions are possible. This is discussed in Example 9 and illustrates how much less simple values at $2 \pi / 3$ are than those at $\pi / 3$. We remark, however, that $\mathrm{Gl}_{2,1}(2 \pi / 3)$ is reducible to one-dimensional polylogarithmic terms [6]. In [1] explicit reductions for all weight four or less polylogarithms are given.

Remark 3. Lewin [20,7.4.3] uses the special case $k=n-$ 2 of (22) to deduce a few small integer evaluations of the log-sine integrals $\operatorname{Ls}_{n}^{(n-2)}(\pi / 3)$ in terms of classical Clausen functions.

In general, we can use (22) recursively to express the log-sine values $\operatorname{Ls}_{n}^{(k)}(\tau)$ in terms of multiple Clausen and Glaisher functions at $\tau$.

Example 8. (22) with $n=5$ and $k=1$ produces

$$
\begin{aligned}
\mathrm{Ls}_{5}^{(1)}(\tau) & =-6 \zeta(4,1)+6 \mathrm{Cl}_{3,1,1}(\tau)+6 \tau \mathrm{Cl}_{2,1,1}(\tau) \\
& +\frac{3}{4} \operatorname{Ls}_{5}^{(3)}(\tau)-\frac{3}{2} \pi \operatorname{Ls}_{4}^{(2)}(\tau)+\frac{3}{4} \pi^{2} \operatorname{Ls}_{3}^{(1)}(\tau) .
\end{aligned}
$$

Applying (22) three more times to rewrite the remaining logsine integrals produces an evaluation of $\operatorname{Ls}_{5}^{(1)}(\tau)$ in terms of multi zeta values and Clausen functions at $\tau$. 


\subsection{Log-sine integrals at $\pi / 3$}

We now apply the general results obtained in Section 5 to the evaluation of log-sine integrals at $\tau=\pi / 3$. Accordingly, we encounter multiple polylogarithms at the basic 6 -th root of unity $\omega:=\exp (i \pi / 3)$. Their real and imaginary parts satisfy various relations and reductions, studied in [4], which allow us to further treat the resulting evaluations. In general, these polylogarithms are more tractable than those at other values because $\bar{\omega}=\omega^{2}$.

Example 9. (Values at $\frac{\pi}{3}$ ) Continuing Example 7 we have

$$
-\mathrm{Ls}_{4}^{(1)}\left(\frac{\pi}{3}\right)=2 \mathrm{Gl}_{3,1}\left(\frac{\pi}{3}\right)+\frac{2}{3} \pi \mathrm{Gl}_{2,1}\left(\frac{\pi}{3}\right)+\frac{19}{6480} \pi^{4} .
$$

Using known reductions from [4] we get:

$$
\mathrm{Gl}_{2,1}\left(\frac{\pi}{3}\right)=\frac{1}{324} \pi^{3}, \quad \mathrm{Gl}_{3,1}\left(\frac{\pi}{3}\right)=-\frac{23}{19440} \pi^{4},
$$

and so arrive at

$$
-\mathrm{Ls}_{4}^{(1)}\left(\frac{\pi}{3}\right)=\frac{17}{6480} \pi^{4} .
$$

Lewin explicitly mentions (28) in the preface to [20] because of its "queer" nature which he compares to some of Landen's curious 18 th century formulas.

Many more reduction besides (27) are known. In particular, the one-dimensional Glaisher and Clausen functions reduce as follows [20]:

$$
\begin{aligned}
\mathrm{Gl}_{n}(2 \pi x) & =\frac{2^{n-1}(-1)^{1+\lfloor n / 2\rfloor}}{n !} B_{n}(x) \pi^{n}, \\
\mathrm{Cl}_{2 n+1}\left(\frac{\pi}{3}\right) & =\frac{1}{2}\left(1-2^{-2 n}\right)\left(1-3^{-2 n}\right) \zeta(2 n+1) .
\end{aligned}
$$

Here, $B_{n}$ denotes the $n$-th Bernoulli polynomial. Further reductions can be derived for instance from the duality result [4, Theorem 4.4]. For low dimensions, we have built these reductions into our program, see Section 6 .

Example 10. (Values of $\operatorname{Ls}_{n}(\pi / 3)$ ) The log-sine integrals at $\pi / 3$ are evaluated by our program as follows:

$$
\begin{aligned}
\mathrm{Ls}_{2}\left(\frac{\pi}{3}\right) & =\mathrm{Cl}_{2}\left(\frac{\pi}{3}\right) \\
-\mathrm{Ls}_{3}\left(\frac{\pi}{3}\right) & =\frac{7}{108} \pi^{3} \\
\mathrm{Ls}_{4}\left(\frac{\pi}{3}\right) & =\frac{1}{2} \pi \zeta(3)+\frac{9}{2} \mathrm{Cl}_{4}\left(\frac{\pi}{3}\right) \\
-\mathrm{Ls}_{5}\left(\frac{\pi}{3}\right) & =\frac{1543}{19440} \pi^{5}-6 \mathrm{Gl}_{4,1}\left(\frac{\pi}{3}\right) \\
\mathrm{Ls}_{6}\left(\frac{\pi}{3}\right) & =\frac{15}{2} \pi \zeta(5)+\frac{35}{36} \pi^{3} \zeta(3)+\frac{135}{2} \mathrm{Cl}_{6}\left(\frac{\pi}{3}\right) \\
-\mathrm{Ls}_{7}\left(\frac{\pi}{3}\right) & =\frac{74369}{326592} \pi^{7}+\frac{15}{2} \pi \zeta(3)^{2}-135 \mathrm{Gl}_{6,1}\left(\frac{\pi}{3}\right)
\end{aligned}
$$

As follows from the results of Section 5 each integral is a multivariable rational polynomial in $\pi$ as well as $\mathrm{Cl}, \mathrm{Gl}$, and zeta values. These evaluations confirm those given in [9, Appendix A] for $\operatorname{Ls}_{3}\left(\frac{\pi}{3}\right), \operatorname{Ls}_{4}\left(\frac{\pi}{3}\right)$, and $\operatorname{Ls}_{6}\left(\frac{\pi}{3}\right)$. Less explicitely, the evaluations of $\operatorname{Ls}_{5}\left(\frac{\pi}{3}\right)$ and $\operatorname{Ls}_{7}\left(\frac{\pi}{3}\right)$ can be recovered from similar results in $[15,9]$ (which in part were obtained using PSLQ; we refer to Section 6 for how our analysis relies on PSLQ).
The first presumed-irreducible value that occurs is

$$
\begin{aligned}
\mathrm{Gl}_{4,1}\left(\frac{\pi}{3}\right) & =\sum_{n=1}^{\infty} \frac{\sum_{k=1}^{n-1} \frac{1}{k}}{n^{4}} \sin \left(\frac{n \pi}{3}\right) \\
& =\frac{3341}{1632960} \pi^{5}-\frac{1}{\pi} \zeta(3)^{2}-\frac{3}{4 \pi} \sum_{n=1}^{\infty} \frac{1}{\left(\begin{array}{c}
2 n \\
n
\end{array}\right) n^{6}} .
\end{aligned}
$$

The final evaluation is described in [4]. Extensive computation suggests it is not expressible as a sum of products of one dimensional Glaisher and zeta values. Indeed, conjectures are made in $[4, \S 5]$ for the number of irreducibles at each depth. Related dimensional conjectures for polylogs are discussed in [26].

\subsection{Log-sine integrals at imaginary values}

The approach of Section 5 may be extended to evaluate log-sine integrals at imaginary arguments. In more usual terminology, these are log-sinh integrals

$$
\operatorname{Lsh}_{n}^{(k)}(\sigma):=-\int_{0}^{\sigma} \theta^{k} \log ^{n-1-k}\left|2 \sinh \frac{\theta}{2}\right| \mathrm{d} \theta
$$

which are related to log-sine integrals by

$$
\operatorname{Lsh}_{n}^{(k)}(\sigma)=(-i)^{k+1} \operatorname{Ls}_{n}^{(k)}(i \sigma) .
$$

We may derive a result along the lines of Theorem 3 by observing that equation (24) is replaced, when $\theta=i t$ for $t>0$, by the simpler

$$
\log \left(1-\mathrm{e}^{-t}\right)=\log \left|2 \sinh \frac{t}{2}\right|-\frac{t}{2} .
$$

This leads to:

ThEOREM 4. For $t>0$, and nonnegative integers $n, k$ such that $n-k \geq 2$,

$$
\begin{aligned}
& \zeta\left(n-k,\{1\}^{k}\right)-\sum_{j=0}^{k} \frac{t^{j}}{j !} \operatorname{Li}_{2+k-j,\{1\}^{n-k-2}}\left(\mathrm{e}^{-t}\right) \\
& \quad=\frac{(-1)^{n+k}}{(n-1) !} \sum_{r=0}^{n-k-1}\left(\begin{array}{c}
n-1 \\
k, r
\end{array}\right)\left(-\frac{1}{2}\right)^{r} \operatorname{Lsh}_{n}^{(k+r)}(t) .
\end{aligned}
$$

Example 11. Let $\rho:=(1+\sqrt{5}) / 2$ be the golden mean. Then, by applying Theorem 4 with $n=3$ and $k=1$,

$$
\begin{aligned}
\operatorname{Lsh}_{3}^{(1)}(2 \log \rho)= & \zeta(3)-\frac{4}{3} \log ^{3} \rho \\
& -\operatorname{Li}_{3}\left(\rho^{-2}\right)-2 \operatorname{Li}_{2}\left(\rho^{-2}\right) \log \rho .
\end{aligned}
$$

This may be further reduced, using $\operatorname{Li}_{2}\left(\rho^{-2}\right)=\frac{\pi^{2}}{15}-\log ^{2} \rho$ and $\operatorname{Li}_{3}\left(\rho^{-2}\right)=\frac{4}{5} \zeta(3)-\frac{2}{15} \pi^{2} \log \rho+\frac{2}{3} \log ^{3} \rho$, to yield the well-known

$$
\operatorname{Lsh}_{3}^{(1)}(2 \log \rho)=\frac{1}{5} \zeta(3) .
$$

The interest in this kind of evaluation stems from the fact that $\log$-sinh integrals at $2 \log \rho$ express values of alternating inverse binomial sums (the fact that log-sine integrals at $\pi / 3$ give inverse binomial sums is illustrated by Example 10 and (30)). In this case,

$$
\operatorname{Lsh}_{3}^{(1)}(2 \log \rho)=\frac{1}{2} \sum_{n=1}^{\infty} \frac{(-1)^{n-1}}{\left(\begin{array}{c}
2 n \\
n
\end{array}\right) n^{3}} .
$$

More on this relation and generalizations can be found in each of $[22,16,4,2]$. 


\section{REDUCING POLYLOGARITHMS}

The techniques described in Sections 3.3 and 5 for evaluating log-sine integrals in terms of multiple polylogarithms usually produce expressions that can be considerably reduced as is illustrated in Examples 3, 4, and 9. Relations between polylogarithms have been the subject of many studies $[3,2]$ with a special focus on (alternating) multiple zeta values $[17,13,26]$ and, to a lesser extent, Clausen values [4].

There is a certain deal of choice in how to combine the various techniques that we present in order to evaluate logsine integrals at certain values. The next example shows how this can be exploited to derive relations among the various polylogarithms involved.

Example 12. For $n=5$ and $k=2$, specializing (19) to $\sigma=\pi$ and $m=1$ yields

$$
\operatorname{Ls}_{5}^{(2)}(2 \pi)=2 \operatorname{Ls}_{5}^{(2)}(\pi)-4 \pi \operatorname{Ls}_{4}^{(1)}(\pi)+4 \pi^{2} \operatorname{Ls}_{3}(\pi) .
$$

By Example 5 we know that this evaluates as $-13 / 45 \pi^{5}$. On the other hand, we may use the technique of Section 3.3 to reduce the log-sine integrals at $\pi$. This leads to

$$
-8 \pi \operatorname{Li}_{3,1}(1)+12 \pi \operatorname{Li}_{4}(1)-\frac{2}{5} \pi^{5}=-\frac{13}{45} \pi^{5} .
$$

In simplified terms, we have derived the famous identity $\zeta(3,1)=\frac{\pi^{4}}{360}$. Similarly, the case $n=6$ and $k=2$ leads to $\zeta(3,1,1)=\frac{3}{2} \zeta(4,1)+\frac{1}{12} \pi^{2} \zeta(3)-\zeta(5)$ which further reduces to $2 \zeta(5)-\frac{\pi^{2}}{6} \zeta(3)$. As a final example, the case $n=7$ and $k=4$ produces $\zeta(5,1)=\frac{\pi^{6}}{1260}-\frac{1}{2} \zeta(3)^{2}$.

For the purpose of an implementation, we have built many reductions of multiple polylogarithms into our program. Besides some general rules, such as (29), the program contains a table of reductions at low weight for polylogarithms at the values 1 and -1 , as well as Clausen and Glaisher functions at the values $\pi / 2, \pi / 2$, and $2 \pi / 3$. These correspond to the polylogarithms that occur in the evaluation of the log-sine integrals at the special values $\pi / 3, \pi / 2,2 \pi / 3, \pi$ which are of particular importance for applications as mentioned in the introduction. This table of reductions has been compiled using the integer relation finding algorithm PSLQ [2]. Its use is thus of heuristic nature (as opposed to the rest of the program which is working symbolically from the analytic results in this paper) and is therefore made optional.

\section{THE PROGRAM}

\subsection{Basic usage}

As promised, we implemented ${ }^{1}$ the presented results for evaluating log-sine integrals for use in the computer algebra systems Mathematica and SAGE. The basic usage is very simple and illustrated in the next example for Mathematica ${ }^{2}$.

Example 13. Consider the log-sine integral $\mathrm{Ls}_{5}^{(2)}(2 \pi)$. The following self-explanatory code evaluates it in terms of polylogarithms:

LsToLi [Ls [5, 2, 2Pi]]

This produces the output $-13 / 45 \pi^{5}$ as in Example 5. As a second example,

$$
\text { -LsToLi [Ls [5, 0, Pi/3]] }
$$

results in the output

$$
1543 / 19440 * \mathrm{Pi}^{\wedge} 5-6 * \mathrm{Gl}[\{4,1\}, \mathrm{Pi} / 3]
$$

which agrees with the evaluation in Example 10. Finally,

$$
\text { LsToLi [Ls }[5,1, \mathrm{Pi}]]
$$

produces

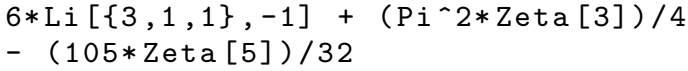

as in Example 3.

\section{Example 14. Computing}

$$
\operatorname{LsToLi}[\mathrm{Ls}[6,3, \mathrm{Pi} / 3]-2 * \operatorname{Ls}[6,1, \mathrm{Pi} / 3]]
$$

yields the value $\frac{313}{204120} \pi^{6}$ and thus automatically proves a result of Zucker [27]. A family of relations between log-sine integrals at $\pi / 3$ generalizing the above has been established in $[22]$.

\subsection{Implementation}

The conversion from log-sine integrals to polylogarithmic values demonstrated in Example 13 roughly proceeds as follows:

- First, the evaluation of $\operatorname{Ls}_{n}^{(k)}(\sigma)$ is reduced to the cases of $0 \leq \sigma \leq \pi$ and $\sigma=2 m \pi$ as described in Section 4.2.

- The cases $\sigma=2 m \pi$ are treated as in Section 3.4 and result in multiple zeta values.

- The other cases $\sigma$ result in polylogarithmic values at $e^{i \sigma}$ and are obtained using the results of Sections 3.3 and 5 .

- Finally, especially in the physically relevant cases, various reductions of the resulting polylogarithms are performed as outlined in Section 6.

\footnotetext{
${ }^{1}$ The packages are freely available for download from http://arminstraub.com/pub/log-sine-integrals

${ }^{2}$ The interface in the case of SAGE is similar but may change slightly, especially as we hope to integrate our package into the core of SAGE.
} 


\subsection{Numerical usage}

The program is also useful for numerical computations provided that it is coupled with efficient methods for evaluating polylogarithms to high precision. It complements for instance the $\mathrm{C}++$ library $\mathrm{lsjk}$ "for arbitrary-precision numeric evaluation of the generalized log-sine functions" described in [15].

Example 15. We evaluate

$$
\begin{aligned}
\mathrm{Ls}_{5}^{(2)}\left(\frac{2 \pi}{3}\right)= & 4 \mathrm{Gl}_{4,1}\left(\frac{2 \pi}{3}\right)-\frac{8}{3} \pi \mathrm{Gl}_{3,1}\left(\frac{2 \pi}{3}\right) \\
& -\frac{8}{9} \pi^{2} \mathrm{Gl}_{2,1}\left(\frac{2 \pi}{3}\right)-\frac{8}{1215} \pi^{5}
\end{aligned}
$$

Using specialized $\operatorname{code}^{3}$ such as [25], the right-hand side is readily evaluated to, for instance, two thousand digit precision in about a minute. The first 1024 digits of the result match the evaluation given in [15]. However, due to its implementation $1 \mathrm{sjk}$ currently is restricted to log-sine functions $\operatorname{Ls}_{n}^{(k)}(\theta)$ with $k \leq 9$.

\section{Acknowledgements.}

We are grateful to Andrei Davydychev and Mikhail Kalmykov for several valuable comments on an earlier version of this paper and for pointing us to relevant publications. We also thank the reviewers for their thorough reading and helpful suggestions.

\section{REFERENCES}

[1] D. Borwein, J. M. Borwein, A. Straub, and J. Wan. Log-sine evaluations of Mahler measures, II. Preprint, March 2011. arXiv:1103.3035.

[2] J. M. Borwein, D. H. Bailey, and R. Girgensohn. Experimentation in Mathematics: Computational Paths to Discovery. A. K. Peters, 1st edition, 2004.

[3] J. M. Borwein, D. M. Bradley, D. J. Broadhurst, and P. Lisoněk. Special values of multiple polylogarithms. Trans. Amer. Math. Soc., 353(3):907-941, 2001. arXiv:math/9910045.

[4] J. M. Borwein, D. J. Broadhurst, and J. Kamnitzer. Central binomial sums, multiple Clausen values, and zeta values. Experimental Mathematics, 10(1):25-34, 2001. arXiv: hep-th/0004153.

[5] J. M. Borwein and R. E. Crandall. Closed forms: what they are and why we care. Notices Amer. Math. Soc., 2010. In press.

[6] J. M. Borwein and A. Straub. Log-sine evaluations of Mahler measures. J. Aust Math. Soc., Mar 2011. arXiv: 1103.3893

[7] D. W. Boyd. Speculations concerning the range of Mahler's measure. Canad. Math. Bull., 24:453-469, 1981.

[8] A. Davydychev and M. Kalmykov. Some remarks on the $\epsilon$-expansion of dimensionally regulated Feynman diagrams. Nuclear Physics B - Proceedings Supplements, 89(1-3):283-288, Oct. 2000. arXiv:hep-th/0005287.

\footnotetext{
${ }^{3}$ The $\mathrm{C}++$ code we used is based on the fast Hölder trans-
} form described in [3], and is available on request.
[9] A. Davydychev and M. Kalmykov. New results for the $\varepsilon$-expansion of certain one-, two- and three-loop Feynman diagrams. Nuclear Physics B, 605:266-318, 2001. arXiv:hep-th/0012189.

[10] A. Davydychev and M. Kalmykov. Massive Feynman diagrams and inverse binomial sums. Nuclear Physics B, 699(1-2):3-64, 2004. arXiv:hep-th/0303162.

[11] A. I. Davydychev. Explicit results for all orders of the $\varepsilon$ expansion of certain massive and massless diagrams. Phys. Rev. D, 61(8):087701, Mar 2000. arXiv: hep-ph/9910224.

[12] W. Fuchs. Two definite integrals (solution to a problem posed by L. Lewin). The American Mathematical Monthly, 68(6):580-581, 1961.

[13] M. E. Hoffman and Y. Ohno. Relations of multiple zeta values and their algebraic expression. J. Algebra, 262:332-347, 2003. arXiv:math/0010140.

[14] M. Kalmykov. About higher order $\epsilon$-expansion of some massive two- and three-loop master-integrals. Nuclear Physics B, 718:276-292, July 2005. arXiv: hep-ph/0503070.

[15] M. Kalmykov and A. Sheplyakov. lsjk - a C++ library for arbitrary-precision numeric evaluation of the generalized log-sine functions. Comput. Phys. Commun., 172(1):45-59, 2005. arXiv: hep-ph/0411100.

[16] M. Kalmykov and O. Veretin. Single scale diagrams and multiple binomial sums. Phys. Lett. B, 483(1-3):315-323, 2000. arXiv:hep-th/0004010.

[17] K. S. Kölbig. Closed expressions for $\int_{0}^{1} t^{-1} \log ^{n-1} t \log ^{p}(1-t) d t$. Mathematics of Computation, 39(160):647-654, 1982.

[18] N. Kurokawa, M. Lalín, and H. Ochiai. Higher Mahler measures and zeta functions. Acta Arithmetica, 135(3):269-297, 2008. arXiv:0908.0171.

[19] L. Lewin. On the evaluation of log-sine integrals. The Mathematical Gazette, 42:125-128, 1958.

[20] L. Lewin. Polylogarithms and associated functions. North Holland, 1981.

[21] D. Maitre. HPL, a Mathematica implementation of the harmonic polylogarithms. Comput. Phys. Commun., 174:222-240, 2006. arXiv:hep-ph/0507152.

[22] Z. Nan-Yue and K. S. Williams. Values of the Riemann zeta function and integrals involving $\log (2 \sinh (\theta / 2))$ and $\log (2 \sin (\theta / 2))$. Pacific J. Math., 168(2):271-289, 1995.

[23] F. W. J. Olver, D. W. Lozier, R. F. Boisvert, and C. W. Clark. NIST Handbook of Mathematical Functions. Cambridge University Press, 2010.

[24] Y. Sasaki. On multiple higher Mahler measures and multiple L values. Acta Arithmetica, 144(2):159-165, 2010.

[25] J. Vollinga and S. Weinzierl. Numerical evaluation of multiple polylogarithms. Comput. Phys. Commun., 167:177, 2005. arXiv:hep-ph/0410259.

[26] S. Zlobin. Special values of generalized polylogarithms. To appear in proceedings of the conference "Diophantine and Analytic Problems in Number Theory", 2007. arXiv:0712.1656.

[27] I. Zucker. On the series $\sum_{k=1}^{\infty}\left(\begin{array}{c}2 k \\ k\end{array}\right)^{-1} k^{-n}$ and related sums. J. Number Theory, 20:92-102, 1985. 